\title{
Distribución y abundancia de Ateles belzebuth E. Geoffroy y Ateles chamek Humboldt (Cebidae: Primates) en la Reserva Nacional Pacaya Samiria, Perú
}

\author{
Distribution and abundance of Ateles belzebuth E. Geoffroy and \\ Ateles chamek Humboldt (Cebidae: Primates) in the Pacaya Samiria \\ National Reserve, Peru
}

Rolando Aquino' y R. E. Bodmer ${ }^{2}$

\begin{abstract}
1. Instituto de Ciencias Biológicas Antonio Raimondi, Facultad de Ciencias Biológicas, Universidad Nacional Mayor de San Marcos. P. O. Box 575, Iquitos, Perú. Telefax 65-265510.

Email: Rolando Aquino: raquinoy2005@yahoo.es

2. Durrell Institute of Conservation and Ecology, University of Kent, Canterbury, England.
\end{abstract}

Presentado: $\quad 01 / 01 / 2006$ Aceptado: $\quad 11 / 03 / 2006$

\begin{abstract}
Resumen
En el presente trabajo se dan a conocer las observaciones sobre el hábitat, las asociaciones inter-específicas, tamaño de grupo y densidad poblacional de las dos especies de Ateles que habitan en la Reserva Nacional Pacaya Samiria. Los datos fueron colectados mediante inventarios y censos por transecto en los periodos de enero de 1997 a febrero de 1999, setiembre -- octubre 2000, agosto -- setiembre, 2002 y enero 2003. En la margen izquierda del Río Samiria, Ateles belzebuth E. Geoffryoy y Ateles chamek Humboldt comparten el hábitat formando grupos mixtos. El tamaño promedio de grupo para $A$. belzebuth fue 5,1 individuos/grupo y para Ateles chamek 7,3 individuos/grupo. La densidad poblacional estimada para $A$. belzebuth fue 1,02 individuos $/ \mathrm{km}^{2}$ y para $A$. chamek 0,51 individuos $/ \mathrm{km}^{2}$. Se discuten y analizan los factores que habrían influido para la drástica reducción de ambas poblaciones.
\end{abstract}

Palabras claves: Reserva Nacional Pacaya Samiria, Ateles, distribución, asociaciones inter-específicas, densidad poblacional.

\section{Abstract}

The study deals with the habitat, inter-especific associations, group size and population density of two Ateles species that inhabit the Pacaya Samiria National Reserve. Data were collected during inventories and transect censuses between january 1997 and february 1999, september - october 2000, august -- september 2002 and January 2003. On the left bank of the Río Samiria, Ateles balzebuth E. Geffroy and Ateles chamek Humboldt share the habitat forming mixed groups. Average group size for $A$. belzebuth was 5,1 individuals/group and for $A$. chamek 7,3 individuals/group. Estimated population density for $A$. belzebuth was 1,02 individuals $/ \mathrm{km}^{2}$ and for $A$. chamek 0,51 individuals $/ \mathrm{km}^{2}$. We discuss and analyze the factors that may have influenced the drastic reduction of both populations.

Keywords: Pacaya Samiria National Reserve, Ateles, distribution, inter-especific associations, population density.

\section{Introducción}

La Reserva Nacional Pacaya Samiria con 2’080,000 ha de extensión y enclavada entre los ríos Ucayali, Marañón y Huallaga es una de las áreas naturales protegidas más importantes del bosque inundable en la Amazonía peruana. En esta reserva constituida principalmente por hábitats de várzea de agua dulce (COREPASA, 1986; INRENA, 2000), habitan 12 especies de primates, entre los que se encuentran A teles belzebuth E. G eoffroy y A teles chamek Humboldt. Estos primates al igual que otros están sujetos a la caza para el consumo de subsistencia (Bodmer, et al., 1997, 1999, Aquino et al., 2000a). No obstante, es muy poco lo que conocemos en referencia a su eto-ecología y dinámica poblacional. La relativa escasez y el difícil contacto incluso no han permitido una clara definición del área de distribución para ambas especies. Los reportes de Neville et al. (1976); Freese et al. (1982) y Soini et al. (1996), mayormente basados en informaciones de guarda parques y otros dan cuenta de la existencia de ambas especies en la reserva. Por su parte, Bodmer et al. (1997) y Aquino et al. (2000a) lograron visualizar algunos grupos de A . belzebuth y A . chamek y proporcionan densidades para el área de caza de Maipuco, Esperanza y San Antonio y cuenca del río Samiria, respectivamente.

La escasa información sobre estos primates nos motivó a la conducción del presenteestudio con el fín de: 1) definir el área de distribución para una y otra especie, 2) determinar el hábitat y 3) estimar la densidad poblacional. Para cumplir con los objetivos propuestos se realizaron exploraciones con fines de inventario y censos por transecto, de enero 1997 a febrero 1999, setiembreoctubre 2000, agosto - septiembre 2002 y enero de 2003. En este trabajo presentamos los resultados obtenidos.

\section{Métodos}

Para los fines de inventario y censos, la cuenca del río Samiria y áreas afines de la Reserva Nacional Pacaya Samiriafue dividida en: 1) área con persistente caza, 2) área con moderada caza y 3) área con ligera caza. En ellas, consideramos 10 estaciones de muestreo distribuidas de la siguiente manera: tres en el área de persistente caza (quebradas de Pinche, Chiric y Parinari), dos en el área de moderada caza (quebradas deWishto Yanayacu y Tacsha cocha) y cinco en el área de ligera caza (quebradas de Armana, 


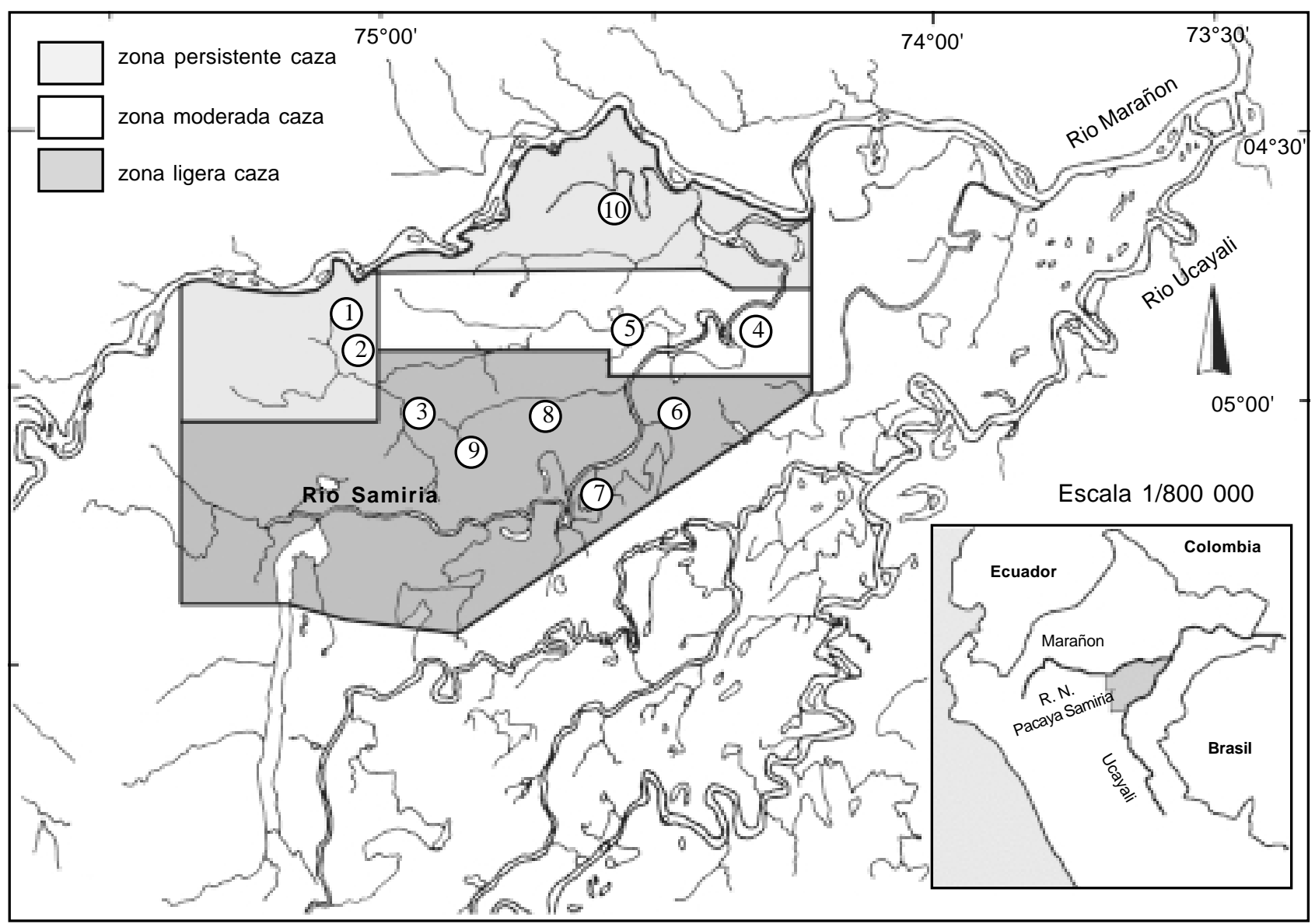

Figura 1. Mapa de la Reserva Nacional Pacaya Samiria mostrando las áreas censadas en la cuenca del río Samiria: 1) quebrada Pinche, 2) quebrada Chiric, 3) quebrada Armana, 4) Tacsha Cocha, 5) quebrada Wishto Yanayacu, 6) quebrada Ungurahui, 7) quebrada Zapote, 8) quebrada Maquizapa, 9) quebrada Guanaico y 10) quebrada Parinari.

Guanaico, Ungurahui, Maquizapa y Zapote) (Fig. 1). Las estaciones de muestreo fueron definidas considerando los antecedentes de la existencia de los A teles a través de reportes, informaciones verbales de guarda parques y cazadores y observaciones directas realizadas en años anteriores por uno de los autores (RA) y otros investigadores.

Para los censos, en cada una de las estaciones de muestreo fueron abiertas de tres a cuatro trochas, cuyas longitudes variaron de 3 a $5 \mathrm{~km}$, dependiendo del tipo de bosque. Por cada una de las trochas, dos observadores provistos de libreta de campo, brújula, binocular, cinta métrica, cintas plásticas y lápiz, se desplazaron a una velocidad de 1,5 km/ hora en las restingas y llanuras y a $0,5 \mathrm{~km} /$ hora en los aguajales. Las actividades censales se realizaron desde las 0700 a $1100 \mathrm{~h}$ y desde las $1500 \mathrm{a} 1800 \mathrm{~h}$ (hora local). Cadavez que hubo contacto con estos primates procedimos al registro de la especie, número de individuos porgrupo y distancia perpendicular del primer animal avistado al transecto. Adicionalmente, anotamos el tipo de bosquey la vegetación dominante. El escaso número de grupos contactados durante los censos no fue suficiente para el estimado de densidades mediante el programa D IST A N CE , por lo que fue usada la fórmula: $D=$ N/ 2LW, donde W es el promedio de la distancia visualizada, L es la longitud total censada y $\mathrm{N}$ es el número de grupos visualizados (Whitesides et al. 1988). La densidad de individuos $/ \mathrm{km}^{2}$ fue obtenida multiplicando el promedio del tamaño de grupo por la densidad de grupos. Un total de 1,287 km de longitud fue censada paraA . belzebuth y $1,552 \mathrm{~km}$ para A . chamek.

\section{Resultados}

\section{Distribución}

Las numerosas exploraciones con fines de inventario y de censos en ambas márgenes del río Samiria nos permite afirmar que A . belzebuth tiene como área de distribución la margen izquierda del río Samiria y comprende desde el río Marañón al Norte, hasta el límite de la reserva al Sur, incluyendo la quebrada Caucho G rande. Al respecto, Kellog y G oldman (1944) ya habían considerado al río Samiria (= río Chamicuro) como parte de la distribución geográfica de esta especie en el Perú.

Por su parte, A . chamek se encuentra habitando en ambas márgenes del río Samiria, por lo tanto tiene por distribución toda la reserva, aún cuando la mayor concentración de su población podría encontrarse en la cuenca del río alto Pacaya (Aquino, obs pers.).

\section{Hábitat}

En la Reserva Nacional Pacaya Samiria, ambas especies parecen tener mayor predisposición por habitar los aguajales. En efecto, de los 13 grupos de A. belzebuth y 6 de A. chamek contactados durante los censos (Tabla 1), solamente tres grupos de A . belzebuth fueron avistados en restingas al borde de aguajales, dos de ellos en la quebrada Wishto Yanayacu comiendo frutos de zapote (A chras zapote) y el tercero en la quebrada Pinche comiendo frutos de granadilla silvestre (Passiflora sp.). Los demás, fueron avistados en aguajales de origen reciente y aguajales semieutrofizados, ambos caracterizados por presentar el piso fango- 
Tabla 1. Grupos de Ateles belzebuth y Ateles chamek observados durante los censos en la cuenca del río Samiria y áreas afines, Reserva Nacional Pacaya Samiria.

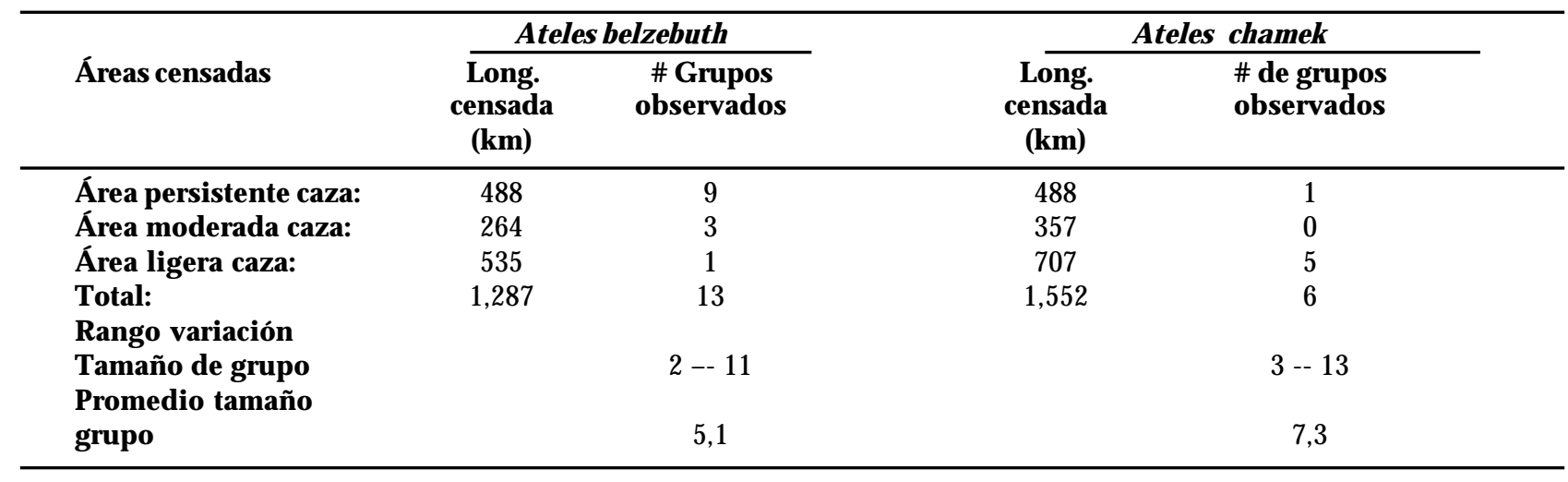

so y cubierto de agua; el primero durante todo el año y el segundo entre seis a ocho meses del año, lo cual probablemente es un limitante para el acceso de cazadores en época de «vaciante» (junio - enero).

\section{Asociaciones inter-específicas}

A . belzebuth y A . chamek, además de compartir el hábitat, también co-existen formando grupos mixtos. Al respecto, en la cuenca del río Samiria se observó en dos oportunidades grupos mixtos, uno en la quebrada Pinche al Norte de la quebrada Yanayacu $G$ rande y otro en la quebrada Guanaico, al Sur de la quebrada Yanayacu $\mathrm{G}$ rande. En el primer caso, el grupo mixto de cuatro ejemplares estuvo conformado por dos de A . belzebuth y dos de A . chamek; mientras que en el segundo, el grupo de 12 individuos estuvo integrado por 4 de A. belzebuth y 8 de A. chamek. En este último, entre los integrantes de A . belzebuth había una hembra adulta sin la banda frontal amarillenta cargando un pequeño infante cuyo pelaje era totalmente negro, lo cual indicaría que entre ambas especies existen cruzamientos.

\section{Tamaño de grupo}

Los grupos contactados de A . belzebuth fluctuaron desde 2 a 11 individuos, cuyo promedio fue estimado en 5,1 y en A . chamek desde 3 a 13 individuos, con un promedio de 7,3 (Tabla 1).

\section{Abundancia y densidad poblacional}

Los resultados nos indican mayor abundancia de A . belzebuth en el área de persistente cazay de A . chamek en el área de ligera caza (Tabla 1), por lo que en lo que concierne a la cuenca del río Samiria y áreas afines, gran parte de la población de A . belzebuth estarían concentradas al Norte de la quebrada Yanayacu $\mathrm{G}$ rande y de A . chamek al Sur de la mencionada quebrada; ambas habitando los aguajales de difícil acceso.

La densidad poblacional en la cuenca del río Samiria fue estimada en 0,2 grupos $/ \mathrm{km}^{2}$ y/ o 1,02 individuos $/ \mathrm{km}^{2}$ para $A$. belz ebuth y 0,06 grupos $/ \mathrm{km}^{2}$ y/ o 0,51 individuos/ $\mathrm{km}^{2}$ para A . chamek (Tabla 2).

Tabla 2. Densidad poblacional estimada para Ateles en la cuenca del río Samiria y áreas afines, Reserva Nacional Pacaya Samiria.

\begin{tabular}{lcc}
\hline Especies & G rupos/ $\mathbf{k m}^{\mathbf{2}}$ & Indiv./ $\mathbf{k m}^{\mathbf{2}}$ \\
\hline Ateles belzebuth & 0,2 & 1,02 \\
A teles chamek & 0,06 & 0,51 \\
\hline
\end{tabular}

\section{Discusión}

En la Reserva Nacional Pacaya Samiria, la mayor predisposición de los A teles por habitar los aguajales podría explicar el escaso o nulo contacto que se tuvo en anteriores censos (con excepción de Aquino et al., 2000a) en los cuales limitaron sus actividades censales a restingas y bosques de galería Al respecto, es muy probable que desde mucho antes de la década de 1970 estos primates ya se hubiesen encontrado refugiados en los aguajales como respuesta a una excesiva caza, causada en primer lugar por el uso como cebo para la captura de felinos (Panthera onc Linnaeus y L eopardus pardalis Linnaeus) cuyas pieles en ese entonces eran cotizadas en el mercado internacional a precios exorbitantes, y en segundo lugar para el consumo de subsistencia debido a su tamaño relativamente grande en comparación a otros primates que habitan en esta reservay por lo agradable de su carne como muchos lo han sostenido.

En referencia a las asociaciones inter-específicas, además de los dos únicos casos registrados entre A . belz ebuth y A . chamek, también fue reportado la asociación de A . chamek con Cacajao calvus ucayalii Thomas en la Reserva Comunal Tamshiyacu Tahuayo (Aquino, 1998). Estos escasos registros indicarían que los A teles usualmente no acostumbran realizar sus actividades en grupos mixtos como ocurre con otras especies (Leonard y Bennet, 1996; Aquino, 1998; Heymann et al. 2002).

El mayor tamaño promedio de grupo deA . chamek respecto al de A . belzebuth; podría mas bien estar relacionado con la pequeña muestra obtenida para A . chamek, aún cuando grupos de hasta 15 individuos fueron registrados para esta especie en la Reserva Comunal Tamshiyacu Tahuayo (Aquino, obs pers.). De otro lado, el tamaño promedio de grupo de ambas especies fue mayor a los reportados por White (1986) para A . chamek en el Parque Nacional Manú y Klein y Klein (1977) para A . belzebuth en Colombia (3,15 y 3,5 respectivamente). Esta diferencia probablemente se debe a los infantes sin locomoción propia que fueron incluidos para los estimados en este estudio.

En este estudio, la densidad estimada para A . belzebuth fue mayor al deA . chamek; también fue mayor a las reportadas para A . chamek en el área de caza de Maipuco, Esperanzay San Antonio (Bodmer et al., 1997), a las de A . belz ebuth y A . chamek en las zonas de persistente cazay ligera caza de la cuenca del nío Samiria (Aquino et al., 2000a) y a las de A . belzebuth en la cuenca del río Pucacuro (A quino etal., 2000b). Estas diferencias podrían estar relacionadas con la realización de censos en aguajales de difícil 
acceso para los cazadores y por ende de mayor concentración de A teles como en los aguajales de Parinari y Wishto Yanayacu. D e otro lado, ambas densidades fueron menores al estimado para A . chamek en el Parque Nacional Manú (White, 1986) y cuenca del río Yavarí (Bodmer et al., 1997); diferencia que podría estar relacionado con la baja presión de caza existente en éstas, en la primera por encontrarse fuertemente resguardado por guarda parques, mientras que en la segunda por su lejanía con respecto a Iquitos, la ciudad más grande en la Amazonía peruana, y por la existencia aún de otros mamíferos de caza relativamente abundantes.

Sorprende quizá el registro del mayor número de grupos de A . belzebuth en el área de persistente caza, es decir, hacia el norte y muy próximo al río Marañón. Lo cual podria explicarse por una relación con la presencia de los aguajales de difícil acceso, que estarían actuando como una barrera casi infranqueable para el ingreso de cazadores furtivos, de otro modo no encontramos explicación para que ambas poblaciones se encuentren habitando casi exclusivamente en este tipo de bosques. También es oportuno precisar que el área que ambas especies comparten el hábitat correspondería a un enclave que se habría originado por el cambio de curso del río Huallaga, quedando parte de la población de A . belzebuth entre los ríos Huallaga y Samiria, muy cerca de la confluencia con el río Marañón, de donde se habrían dispersado hacia el sur.

\section{Agradecimientos}

Nuestra gratitud al desaparecido Dr. José López Parodi, quien en vida fue Director del Programa Integral de D esarrollo y Conservación Pacaya Samiria, AIF-WWF/ DK, al M.S. Pablo Puertas, Director Interino del Programa Integral de D esarrollo y Conservación Pacaya Samiria, AIF-WWF/ DK y al Consejo Superior de Investigaciones (CSI) de la Universidad Nacional Mayor de San Marcos, por habernos facilitado el financiamiento, sin los cuales no hubiera sido posible la ejecución del presente estudio. Nuestro reconocimiento al Instituto Nacional de Recursos Naturales (INRENA) por el permiso otorgado para el ingreso a la citada reserva. Hacemos extensivo nuestra gratitud al personal de la Jefatura Pacaya Samiria por brindarnos el apoyo logístico y a los moradores de la comunidad de Parinari, por su activa participación durante los censos en aguajales.

\section{Literatura citada}

Aquino, R. 1998. Some observations on the ecology of Cacajao calvus ucayalii in the Peruvian Amazon. Primate Conservation 18: $21-24$.

Aquino, R.; R. E. Bodmer y G. Gil. 2000a. Impacto de la caza en poblaciones de primates de la cuenca del río Samiria, Reserva Nacional Pacata Samiria. En: F. San Martín y M. García (ed.): La Primatología en el Perú. Lima, Perú, Vol. II, $81-91$.
Aquino, R.; R. E. Bodmer y E. Pezo. 2000b. Evaluación de primates en la cuenca del río Pucacuro, Amazonia Peruana. En: F. San Martín y M. García (ed.): La Primatología en el Perú. Lima, Perú, Vol. II, 92 - 100.

Bodmer, R. E.; R. Aquino y P. Puertas. 1997. Alternativas de manejo para la Reserva Nacional Pacaya Samiria: Un análisis sobre el uso sostenible de la caza. En: T. Fang, R. E. Bodmer, R. Aquino y M. Valqui (ed.): Manejo de Fauna Silvestre en la Amazonia. La Paz, Bolivia, 65 - 74.

Bodmer, R.E.; C. Allen; J. Penn; R. Aquino y C. Reyes. 1999. Evaluación del uso sostenible de la fauna silvestre en la Reserva Nacional Pacaya Samiria. Documento de Trabajo América Verde 4b, The Nature Conservancy, Arlington, USA.

COREPASA, 1986. Plan Maestro de la Reserva Nacional Pacaya Samiria. Editorial e Imprenta DESA, Iquitos, Perú.

INRENA, 2000. Plan Maestro de la Reserva Nacional Pacaya Samiria. Loreto, Perú.

Freese, C.; P. Heltne; N. Castro \& G. Whitesides. 1982. Patterns and determinants of monkey densities in Peru and Bolivia, with notes on distribution. Int. J. Primatol. 3(1): 53 -90 .

Heymann, E.W.; F. Encarnación; y J. E. Canaquiri. 2002. Primates of the río Curaray, northern Peruvian Amazon. Int. J. Primatol. 23(1):191 - 201.

Kellog, R. \& E. Goldman, E.1944. Review of spider monkeys. Proc. U.S. National Museum 96: 1 - 45.

Klein, L.L. \& L. J. Klein. 1977. Feeding behavior of the Colombian spider monkey, Ateles belzebuth. En: T.H. Clutton-Brock (ed.): Primate Ecology: Studies of feeding and ranging behavior in lemurs, monkeys and apes. New York, Academic Press, 153 - 181.

Leonard, Z. \& C. Bennet. 1996. Associative behavior of Cacajao calvus ucayalii with other primate species in Amazonia Peru. Primates 37(2): $227-230$.

Neville, M.; N. Castro; A. Mármol \& J. Revilla. 1976. Censusing primate population in the reserved area of the Pacaya and Samiria rivers, Department Loreto, Peru. Primates 17 (2): 151 - 181.

Soini, P.; L. Sicchard; A. Fachín; R. Pezo y M. Chumbe. 1996. Una evaluación de la fauna silvestre y su aprovechamiento de la Reserva Nacional Pacaya Samiria, Perú. IIAP. Documento Técnico No. 24.

White, F. 1986. Census and preliminary observation on the ecology of black-faced black spider monkey (Ateles paniscus chamek) in Manu National Park, Peru. Am. J. Primatol. 11: 125 - 132.

Whitesides, G. H.; J. F. Oates; S. M. Green y R. P. Kluberdanz. 1988. Estimating primate densities from transects in African rain forest: A comparison of techniques. J. Anim. Ecol. 57: 345 -367. 